\title{
CONNECTION COEFFICIENTS, MATCHINGS, MAPS AND COMBINATORIAL CONJECTURES FOR JACK SYMMETRIC FUNCTIONS
}

\author{
I. P. GOULDEN AND D. M. JACKSON
}

\begin{abstract}
A power series is introduced that is an extension to three sets of variables of the Cauchy sum for Jack symmetric functions in the Jack parameter $\alpha$. We conjecture that the coefficients of this series with respect to the power sum basis are nonnegative integer polynomials in $b$, the Jack parameter shifted by 1 . More strongly, we make the Matchings-Jack Conjecture, that the coefficients are counting series in $b$ for matchings with respect to a parameter of nonbipartiteness. Evidence is presented for these conjectures and they are proved for two infinite families.

The coefficients of a second series, essentially the logarithm of the first, specialize at values 1 and 2 of the Jack parameter to the numbers of hypermaps in orientable and locally orientable surfaces, respectively. We conjecture that these coefficients are also nonnegative integer polynomials in $b$, and we make the Hypermap-Jack Conjecture, that the coefficients are counting series in $b$ for hypermaps in locally orientable surfaces with respect to a parameter of nonorientability.
\end{abstract}

\section{INTRODUCTION}

Let $J_{\theta}(\mathbf{x} ; \alpha)$ be the Jack symmetric function of $\mathbf{x}=\left(x_{1}, x_{2}, \ldots\right)$, indexed by a partition $\theta$ and an indeterminate $\alpha$, with norm $\sqrt{\left\langle J_{\theta}, J_{\theta}\right\rangle_{\alpha}}$. Let $\mathcal{P}$ be the set of all partitions, with the null partition adjoined, and let $|\theta|$ be the integer partitioned by $\theta$. In this paper we study the series

$$
\Phi(\mathbf{x}, \mathbf{y}, \mathbf{z} ; t, \alpha)=\sum_{\theta \in \mathcal{P}} \frac{1}{\left\langle J_{\theta}, J_{\theta}\right\rangle_{\alpha}} J_{\theta}(\mathbf{x} ; \alpha) J_{\theta}(\mathbf{y} ; \alpha) J_{\theta}(\mathbf{z} ; \alpha) t^{|\theta|},
$$

that is associated with matchings and with the connection coefficients of the double coset algebra of the hyperoctahedral group, and the related series

$$
\Psi(\mathbf{x}, \mathbf{y}, \mathbf{z} ; t, \alpha)=\alpha t \frac{\partial}{\partial t} \log \Phi(\mathbf{x}, \mathbf{y}, \mathbf{z} ; t, \alpha),
$$

that is associated with 2-cell embeddings of maps in locally orientable surfaces.

Most of our attention is focussed on $\Phi$, and its properties. However, since $\Psi$ offers substantial evidence of the significance of these two series, we will digress briefly to describe its combinatorial background, and then return to $\Psi$ again only in the final section. The major portion of this paper therefore can be read independently of the combinatorial background of $\Psi$.

Received by the editors November 27, 1994.

1991 Mathematics Subject Classification. Primary 05E05, 05A15, 57M15. 
The Jack symmetric functions specialize at $\alpha=2$ to zonal polynomials and at $\alpha=1$, suitably scaled, to Schur symmetric functions. They were introduced by the statistician Henry Jack [11], and have been the subject of substantial recent interest in combinatorial theory (e.g. $[3,8,9,14,16,18]$ ), especially motivated by Macdonald's [16] presentation at the 1985 Durham Symposium. In the context of the uniform algebraic treatment applied to symmetric functions (see, for example, [17]) as a whole, $\Phi$ can be regarded as an extension of the Cauchy sum (10) to a third set of indeterminates. Another instance of such a sum is given in $[1,2]$. Indirectly, the conjectures about $\Phi$ and $\Psi$ that are described below offer some support for the possibility that $\alpha$, the Jack parameter, may itself have a combinatorial role.

1.1. Combinatorial background of $\Psi$ : hypermaps. The study of $\Psi$ originates in recent work on hypermaps reported in $[6,12,13]$, where the specializations of $\Psi$ at $\alpha=1$ and 2 are identified as generating series for hypermaps in orientable and locally orientable surfaces, respectively.

Recall that a locally orientable surface is closed, without boundary and either orientable or nonorientable. A map in a locally orientable surface is a 2-cell embedding of a graph in the surface, so the deletion of the edges of the graph decomposes the surface into regions homeomorphic to open discs, corresponding to faces of the map. The vertices and edges of the graph are the vertices and edges of the map. A hypermap is a face two-coloured map, so each edge separates faces of different colours. The hyperedges of the hypermap are the faces of one (specified) colour, and the faces are the faces of the other colour. The face distribution is a partition whose parts list the degrees of the faces, the hyperedge distribution lists the degrees of the hyperedges and the vertex distribution lists the number of hyperedges incident with the vertices. A hypermap is rooted by distinguishing a unique side and end of an edge bounding a hyperedge. Regarded as a hypermap, a map corresponds to a hypermap in which all hyperedges have degree two (and these can then be identified to give a single edge), so the embedding theory of maps is recoverable from the embedding theory for hypermaps.

Let $m_{\mu \nu}^{\lambda}$ and $l_{\mu \nu}^{\lambda}$, respectively, be the numbers of rooted hypermaps in orientable and locally orientable surfaces, with vertex distribution $\mu$, face distribution $\nu$ and hyperedge distribution $\lambda$. The generating series for $m_{\mu \nu}^{\lambda}$ has been investigated extensively by Jackson and Visentin $[12,13]$, through the combinatorial relationship between orientable maps and permutations given by rotation systems, while the generating series for $l_{\mu \nu}^{\lambda}$ has recently been determined in [6] by using a combinatorial relationship between locally orientable maps and matchings. These generating series are also called genus series.

The power sum basis of the ring of symmetric functions affords a convenient basis for the presentation of the genus series. Let $p_{\lambda}$ be the power sum symmetric function indexed by $\lambda$.

Theorem 1.1. The genus series for hypermaps in orientable and locally orientable surfaces, respectively, are given by

$$
\begin{aligned}
\text { 1) } & \sum_{n \geq 1} t^{n} \sum_{\lambda, \mu, \nu \vdash n} m_{\mu \nu}^{\lambda} p_{\lambda}(\mathbf{x}) p_{\mu}(\mathbf{y}) p_{\nu}(\mathbf{z})=\Psi(\mathbf{x}, \mathbf{y}, \mathbf{z} ; t, 1), \\
\text { 2) } & \sum_{n \geq 1} t^{n} \sum_{\lambda, \mu, \nu \vdash n} l_{\mu \nu}^{\lambda} p_{\lambda}(\mathbf{x}) p_{\mu}(\mathbf{y}) p_{\nu}(\mathbf{z})=\Psi(\mathbf{x}, \mathbf{y}, \mathbf{z} ; t, 2) .
\end{aligned}
$$


The parts of Theorem 1.1 were originally stated directly in terms of Schur functions and zonal polynomials. The purpose of this paper is to study the consequences of the above uniform identifications of the right-hand sides of this theorem, as different specializations of the series $\Psi$.

In view of these results, we define the hypermap series $h_{\mu \nu}^{\lambda}(b)$, by

$$
\Psi(\mathbf{x}, \mathbf{y}, \mathbf{z} ; t, 1+b)=\sum_{n \geq 1} t^{n} \sum_{\lambda, \mu, \nu \vdash n} h_{\mu \nu}^{\lambda}(b) p_{\lambda}(\mathbf{x}) p_{\mu}(\mathbf{y}) p_{\nu}(\mathbf{z}) .
$$

The theory of Jack symmetric functions then implies that the hypermap series are, in general, rational functions of $b$. However, in a computational investigation, we found that the hypermap series appeared actually to be polynomials in $b$, and in Section 6 of this paper, we conjecture (the Hypermap-Jack Conjecture) that

$$
h_{\mu \nu}^{\lambda}(b)=\sum_{M} b^{\vartheta(M)}
$$

where the summation is over hypermaps $M$ in locally orientable surfaces with vertex distribution $\mu$, face distribution $\nu$ and hyperedge distribution $\lambda$, with respect to a combinatorial statistic $\vartheta$ correlated with the nonorientability of hypermaps.

1.2. Combinatorial background of $\Phi$ : matchings. In obtaining evidence for the above conjecture, we found $\Psi$ to be mathematically tractable only through its relationship (2) with the series $\Phi$. Moreover, the combinatorial derivations of Theorem 1.1 given in $[6,12,13]$ involve the specialisations of $\Phi$ at $\alpha=1,2$ in an essential way, as the generating series for connection coefficients of two commutative algebras. Thus we were led to a study of $\Phi$ itself in the power sum basis, and define the connection series $c_{\mu \nu}^{\lambda}(b)$, by

$$
\Phi(\mathbf{x}, \mathbf{y}, \mathbf{z} ; t, 1+b)=\sum_{n \geq 1} t^{n} \sum_{\lambda, \mu, \nu \vdash n} \frac{c_{\mu \nu}^{\lambda}(b)}{(1+b)^{l(\lambda)}} \frac{\left|\mathcal{C}_{\lambda}\right|}{n !} p_{\lambda}(\mathbf{x}) p_{\mu}(\mathbf{y}) p_{\nu}(\mathbf{z}),
$$

where $l(\lambda)$ is the number of parts of $\lambda$ and $\mathcal{C}_{\lambda}$ is the conjugacy class of the symmetric group indexed by $\lambda$. Note that the coefficients in this basis are scaled by a nontrivial function of $\lambda$. Again the theory of Jack symmetric functions implies that the connection series are rational functions of $b$, yet computationally we found that they appeared to be polynomials. A combinatorial investigation of the connection series was then carried out, as suggested by the matchings representation of hypermaps used in [6]. Thus, in Section 4 of this paper we conjecture (the Matchings-Jack Conjecture) that

$$
c_{\mu \nu}^{\lambda}(b)=\sum_{\delta} b^{\mathrm{wt}_{\lambda}(\delta)}
$$

where the summation is over a particular subset of matchings $\delta$ and $\mathrm{wt}_{\lambda}$ is a combinatorial statistic correlated with the nonbipartiteness of matchings.

1.3. Organisation of the paper. Section 2 gives the properties of the Jack symmetric functions that will be required, and the definitions of two commutative subalgebras of the group algebra of the symmetric group and their connection coefficients. The two algebras are the class algebra of the symmetric group and the Hecke algebra associated with the hyperoctahedral group. In Section 3, we demonstrate the relationship between the connection series $c_{\mu \nu}^{\lambda}(b)$ and the connection coefficients of the two algebras. We obtain some explicit marginal sums for the 
connection series and some explicit evaluations of it for certain values of $\lambda$. These are obtained by extensive use of the properties of Jack symmetric functions that are given in Section 2. In Section 4 we give the relationship between the connection coefficients of two algebras and classes of matchings, and we state the MatchingsJack Conjecture. In Section 5 we exhibit two combinatorial statistics for matchings associated with two particular choices of $\lambda$. We prove that the generating series for matchings with respect to these statistics are the connection series explicitly evaluated in Section 3, thus establishing the Matchings-Jack Conjecture for two particular infinite subfamilies of matchings. In Section 6 we turn our attention to $\Psi$ and its coefficients, the hypermap series, and we state the Hypermap-Jack Conjecture. Evidence for the truth of this conjecture is presented.

\section{BACKGROUND}

2.1. Partitions. If $\lambda=\left[\lambda_{1}, \lambda_{2}, \ldots\right]$ is a weakly decreasing sequence of nonnegative integers, then $\lambda$ is a partition of $n=\lambda_{1}+\lambda_{2}+\cdots$, and we write $|\lambda|=n$, or $\lambda \vdash n$. The number of nonzero $\lambda_{i}$ in $\lambda$ is denoted by $l(\lambda)$. The set of all partitions (including the single, empty partition of 0 ) is denoted by $\mathcal{P}$. If $\mu=\left[\mu_{1}, \mu_{2}, \ldots\right]$ is another partition with $|\mu|=|\lambda|$, then the partial order $\leq$ is defined for $\mathcal{P}$ by: $\mu \leq \lambda$ whenever $\mu_{1}+\cdots+\mu_{i} \leq \lambda_{1}+\cdots+\lambda_{i}$ for all $i \geq 1$. The partition $2 \lambda$ is given by $\left[2 \lambda_{1}, 2 \lambda_{2}, \ldots\right]$. The conjugate partition to $\lambda$ is given by $\lambda^{\prime}=\left(\lambda_{1}^{\prime}, \lambda_{2}^{\prime}, \ldots\right)$ where $\lambda_{j}^{\prime}$ is the number of $i$ such that $\lambda_{i} \geq j$ for $j \geq 1$.

A partition $\lambda$ is identified with its Ferrers diagram

$$
\lambda=\left\{(i, j): 1 \leq i \leq l(\lambda), 1 \leq j \leq \lambda_{i}\right\} .
$$

For each lattice point $x=(s, t)$ in $\lambda$,

$$
\begin{aligned}
a_{\lambda}(x) & =|\{(s, j) \in \lambda: j>t\}| \text { is the arm-length of } x, \\
l_{\lambda}(x) & =|\{(i, t) \in \lambda: i>s\}| \text { is the leg-length of } x, \\
h_{\lambda}(x) & =a_{\lambda}(x)+l_{\lambda}(x)+1 \text { is the hook-length of } x .
\end{aligned}
$$

The product of all the hook-lengths in $\lambda$ is denoted by

$$
H_{\lambda}=\prod_{x \in \lambda} h_{\lambda}(x)
$$

When convenient, we also write $\lambda=\left[1^{a_{1}} 2^{a_{2}} \ldots\right]$ where $a_{i}$ is the number of times that $i$ appears as a part in $\lambda$.

2.2. Jack symmetric functions. The following notation is well established (see Macdonald [15] for more details). The symmetric functions in $\mathbf{x}=\left(x_{1}, x_{2}, \ldots\right)$, indexed by partition $\lambda \in \mathcal{P}$ are denoted by: $m_{\lambda}(\mathbf{x})$, monomial symmetric function; $p_{\lambda}(\mathbf{x})$, power sum; $s_{\lambda}(\mathbf{x})$, Schur function; $Z_{\lambda}(\mathbf{x})$, zonal polynomial; $J_{\lambda}(\mathbf{x} ; \alpha)$, Jack symmetric function. We will also consider symmetric functions of $\mathbf{y}=\left(y_{1}, y_{2}, \ldots\right)$, $\mathbf{z}=\left(z_{1}, z_{2}, \ldots\right)$, and corresponding finite sets $\mathbf{x}_{n}=\left(x_{1}, \ldots, x_{n}\right), \mathbf{y}_{n}$ and $\mathbf{z}_{n}$, but suppress the arguments where there is no ambiguity.

An inner product $\langle\cdot, \cdot\rangle_{\alpha}$ is defined on the vector space of symmetric functions of bounded degree, with coefficients that are rational functions in the indeterminate $\alpha$ over the rationals, by

$$
\left\langle p_{\lambda}, p_{\mu}\right\rangle_{\alpha}=\delta_{\lambda \mu} \frac{\left|\mathcal{C}_{\lambda}\right|}{|\lambda| !} \alpha^{l(\lambda)}
$$


Macdonald [15] has proved that the Jack symmetric functions are uniquely determined by the following three properties:

$$
\begin{aligned}
& \text { (I) }\left\langle J_{\lambda}, J_{\mu}\right\rangle_{\alpha}=0 \quad \text { for } \quad \lambda \neq \mu, \\
& \text { (II) } \quad\left[m_{\mu}\right] J_{\lambda}=0 \text { unless } \quad \mu \leq \lambda \text {, } \\
& \text { (III) } \left.\left[m_{\left[1^{n}\right]}\right] J_{\lambda}=n \text { ! for all } \lambda \vdash n,\right\}
\end{aligned}
$$

where $[\mathrm{A}] \mathrm{B}$ denotes the coefficient of $\mathrm{A}$ in $\mathrm{B}$. For the purposes of the present work, the natural basis for the Jack symmetric functions is the power sum (symmetric function) basis. The first few Jack symmetric functions, expanded with respect to this basis, are

$$
\begin{aligned}
J_{[1]} & =p_{1}, \\
J_{[2]} & =p_{1}^{2}+\alpha p_{2}, \\
J_{\left[1^{2}\right]} & =p_{1}^{2}-p_{2}, \\
J_{[3]} & =p_{1}^{3}+3 \alpha p_{2} p_{1}+2 \alpha^{2} p_{3}, \\
J_{[21]} & =p_{1}^{3}+(\alpha-1) p_{2} p_{1}-\alpha p_{3}, \\
J_{\left[1^{3}\right]} & =p_{1}^{3}-3 p_{2} p_{1}+2 p_{3} .
\end{aligned}
$$

In general, properties (6) together with basic facts about symmetric functions, imply that the coefficients in these expansions are rational functions of $\alpha$ over the rationals. It has been conjectured that the coefficients are in fact polynomials in $\alpha$ over the integers. This conjecture remains open, and the reader is directed to Macdonald [16] and Hanlon [8] for a discussion.

2.3. Technical results for Jack symmetric functions. The technical results about $J_{\lambda}$ that are essential to the argument are grouped below, and stated without proof. These, together with many other results and a systematic development, have been given by Stanley [18].

Specialization

$$
\left.\begin{array}{rl}
J_{\lambda}(\mathbf{x} ; 1) & =H_{\lambda} s_{\lambda}(\mathbf{x}), \\
J_{\lambda}(\mathbf{x} ; 2) & =Z_{\lambda}(\mathbf{x}) .
\end{array}\right\}
$$

Single-part partition

(8)

$$
\left.\begin{array}{rlrl}
J_{[n]}(\mathbf{x} ; \alpha) & =\sum_{\lambda \vdash n}\left|\mathcal{C}_{\lambda}\right| \alpha^{n-l(\alpha)} p_{\lambda}(\mathbf{x}), & \\
J_{\lambda}((t, 0,0, \ldots) ; \alpha) & =0, & \\
& =(1+\alpha)(1+2 \alpha) \cdots(1+(n-1) \alpha) t^{n}, & \text { if } \quad \lambda=[n] .
\end{array}\right\}
$$

Norm

$$
\left.\begin{array}{rl}
\left\langle J_{\lambda}, J_{\lambda}\right\rangle_{\alpha} & =\prod_{x \in \lambda}\left(\alpha a_{\lambda}(x)+l_{\lambda}(x)+\alpha\right)\left(\alpha a_{\lambda}(x)+l_{\lambda}(x)+1\right), \\
\left\langle J_{[n]}, J_{[n]}\right\rangle_{\alpha} & =n ! \alpha^{n}(1+\alpha)(1+2 \alpha) \cdots(1+(n-1) \alpha) .
\end{array}\right\}
$$

Cauchy Identity

$$
\sum_{\theta \in \mathcal{P}} \frac{1}{\left\langle J_{\theta}, J_{\theta}\right\rangle_{\alpha}} J_{\theta}(\mathbf{x} ; \alpha) J_{\theta}(\mathbf{y} ; \alpha)=\prod_{i, j \geq 1}\left(1-x_{i} y_{j}\right)^{-1 / \alpha} .
$$

A power sum coefficient

$$
\left[p_{2} p_{1}^{n-2}\right] J_{\lambda}=\alpha \sum_{i}\left(\begin{array}{c}
\lambda_{i} \\
2
\end{array}\right)-\sum_{i}\left(\begin{array}{c}
\lambda_{i}^{\prime} \\
2
\end{array}\right), \quad \text { for } \lambda \vdash n .
$$


As eigenfunctions

(12)

$$
D\left(\mathbf{x}_{m} ; \alpha\right) J_{\lambda}\left(\mathbf{x}_{m} ; \alpha\right)=\left\{\alpha \sum_{i}\left(\begin{array}{c}
\lambda_{i} \\
2
\end{array}\right)-\sum_{i}\left(\begin{array}{c}
\lambda_{i}^{\prime} \\
2
\end{array}\right)+(n-1)|\lambda|\right\} J_{\lambda}\left(\mathbf{x}_{m} ; \alpha\right),
$$

where, for a finite set of indeterminates, the generalized Laplace-Beltrami Operator $D\left(\mathbf{x}_{m} ; \alpha\right)$ is defined by

$$
D\left(\mathbf{x}_{m} ; \alpha\right)=\frac{\alpha}{2} \sum_{i=1}^{m} x_{i}^{2} \frac{\partial^{2}}{\partial x_{i}^{2}}+\sum_{i=1}^{m} \sum_{j \neq i} \frac{x_{i}^{2}}{x_{i}-x_{j}} \frac{\partial}{\partial x_{i}} .
$$

An immediate consequence of these is the next result, specializing $\Phi$ to forms involving Schur functions and zonal polynomials.

Proposition 2.1.

$$
\begin{aligned}
& \text { 1) } \Phi(\mathbf{x}, \mathbf{y}, \mathbf{z} ; t, 1)=\sum_{\theta \in \mathcal{P}} H_{\theta} s_{\theta}(\mathbf{x}) s_{\theta}(\mathbf{y}) s_{\theta}(\mathbf{z}) t^{|\theta|} \\
& \text { 2) } \Phi(\mathbf{x}, \mathbf{y}, \mathbf{z} ; t, 2)=\sum_{\theta \in \mathcal{P}} \frac{1}{H_{2 \theta}} Z_{\theta}(\mathbf{x}) Z_{\theta}(\mathbf{y}) Z_{\theta}(\mathbf{z}) t^{|\theta|} .
\end{aligned}
$$

Proof. 1) We have $\left\langle J_{\theta}, J_{\theta}\right\rangle_{1}=H_{\theta}^{2}$ from (9), and the result follows from (7).

2) We have $\left\langle J_{\theta}, J_{\theta}\right\rangle_{2}=H_{2 \theta}$ from (9), and the result follows from (7).

2.4. The two commutative subalgebras and their connection coefficients. The two commutative subalgebras of the group algebra of the symmetric group that are needed are defined below. The essential results are stated without proof, and for a more complete development the reader is directed to Hanlon, Stanley and Stembridge [10].

1) The class algebra: Let $\boldsymbol{C}_{\lambda} \in \mathbb{C S}_{n}$ be the formal sum of the elements in the conjugacy class $\mathcal{C}_{\lambda}$ of $\mathfrak{S}_{n}$, for $\lambda \vdash n$. Then $\left\{\mathcal{C}_{\lambda}: \lambda \vdash n\right\}$ is a basis for the class algebra, a commutative subalgebra of $\mathbb{C S}_{n}$, with connection coefficients

$$
a_{\mu \nu}^{\lambda}=\left[\mathrm{C}_{\lambda}\right] \mathrm{C}_{\mu} \mathrm{C}_{\nu} \text {. }
$$

2) The double coset algebra: Consider the hyperoctahedral group $\mathcal{B}_{n}$ as a subgroup of the symmetric group $\mathfrak{S}_{2 n}$. The double cosets of $\mathfrak{S}_{2 n}$ with $\mathcal{B}_{n}$ have the partitions of $n$ as a natural index. Let $\mathcal{K}_{\lambda}$ be the double coset indexed by $\lambda \vdash n$ and let $\mathrm{K}_{\lambda} \in \mathbb{C S}_{2 n}$ be the formal sum of the elements of $\mathcal{K}_{\lambda}$. Then $\left\{\mathrm{K}_{\lambda}: \lambda \vdash n\right\}$ is a basis for the double coset algebra, a commutative subalgebra of $\mathbb{C S}_{2 n}$, with connection coefficients

$$
b_{\mu \nu}^{\lambda}=\left[\mathrm{K}_{\lambda}\right] \mathrm{K}_{\mu} \mathrm{K}_{\nu} .
$$

The generating series for these connection coefficients with respect to the power sum symmetric function basis are given in the following theorem.

Theorem 2.2.

1) $\Phi(\mathbf{x}, \mathbf{y}, \mathbf{z} ; t, 1)=1+\sum_{n \geq 1} t^{n} \sum_{\lambda, \mu, \nu \vdash n} a_{\mu \nu}^{\lambda} \frac{\left|\mathcal{C}_{\lambda}\right|}{n !} p_{\lambda}(\mathbf{x}) p_{\mu}(\mathbf{y}) p_{\nu}(\mathbf{z})$,

2) $\Phi(\mathbf{x}, \mathbf{y}, \mathbf{z} ; t, 2)=1+\sum_{n \geq 1} t^{n} \sum_{\lambda, \mu, \nu \vdash n} \frac{b_{\mu \nu}^{\lambda}}{n ! 2^{n}} \frac{\left|\mathcal{C}_{\lambda}\right|}{n ! 2^{l(\lambda)}} p_{\lambda}(\mathbf{x}) p_{\mu}(\mathbf{y}) p_{\nu}(\mathbf{z})$. 
Proof. 1) From [6] (see also [12, 13]),

$$
1+\sum_{n \geq 1} t^{n} \sum_{\lambda, \mu, \nu \vdash n} a_{\mu \nu}^{\lambda} \frac{\left|\mathcal{C}_{\lambda}\right|}{n !} p_{\lambda}(\mathbf{x}) p_{\mu}(\mathbf{y}) p_{\nu}(\mathbf{z})=\sum_{\theta \in \mathcal{P}} H_{\theta} s_{\theta}(\mathbf{x}) s_{\theta}(\mathbf{y}) s_{\theta}(\mathbf{z}) t^{|\theta|} .
$$

The result follows from Proposition 2.1(1).

2) From [6],

$$
1+\sum_{n \geq 1} t^{n} \sum_{\lambda, \mu, \nu \vdash n} \frac{b_{\mu \nu}^{\lambda}}{n ! 2^{n}} \frac{\left|\mathcal{C}_{\lambda}\right|}{n ! 2^{l(\lambda)}} p_{\lambda}(\mathbf{x}) p_{\mu}(\mathbf{y}) p_{\nu}(\mathbf{z})=\sum_{\theta \in \mathcal{P}} \frac{1}{H_{2 \theta}} Z_{\theta}(\mathbf{x}) Z_{\theta}(\mathbf{y}) Z_{\theta}(\mathbf{z}) t^{|\theta|}
$$

The result follows from Proposition 2.1(2).

\section{The CONNECTION SERIES AND A POSITIVITy CONJECTURE}

3.1. The connection series. From (5), the generating series for the connection series $c_{\mu \nu}^{\lambda}(b)$ is

$$
\Phi(\mathbf{x}, \mathbf{y}, \mathbf{z} ; t, 1+b)=1+\sum_{n \geq 1} t^{n} \sum_{\lambda, \mu, \nu \vdash n} \frac{c_{\mu \nu}^{\lambda}(b)}{(1+b)^{l(\lambda)}} \frac{\left|\mathcal{C}_{\lambda}\right|}{n !} p_{\lambda}(\mathbf{x}) p_{\mu}(\mathbf{y}) p_{\nu}(\mathbf{z}) .
$$

The reasons for writing the Jack parameter as $1+b$ will become clear in Section 4 , where $b$ is conjectured to have combinatorial meaning. From the basic properties of Jack symmetric functions, in general $c_{\mu \nu}^{\lambda}(b)$ is a rational function of $b$ for all $\lambda, \mu, \nu \vdash n$. The relationship of the connection series to the connection coefficients of the two algebras, that has been alluded to in the Introduction, is given next and follows immediately from (16) and Theorem 2.2.

Proposition 3.1. For $\lambda, \mu, \nu \vdash n$,

$$
\begin{aligned}
& \text { 1) } c_{\mu \nu}^{\lambda}(0)=a_{\mu \nu}^{\lambda}, \\
& \text { 2) } \quad c_{\mu \nu}^{\lambda}(1)=\frac{b_{\mu \nu}^{\lambda}}{2^{n} n !} .
\end{aligned}
$$

3.2. Specific evaluations. Although explicit information about $c_{\mu \nu}^{\lambda}(b)$ is not easy to obtain in general, it is possible to determine some marginal sums and explicit evaluations using the technical results for Jack symmetric functions given in Section 2.3. These will furnish evidence in support of the subsequent conjectures.

a) The marginal sums. We first obtain marginal sums over $\mu$ (or $\nu$ ), and marginal sums over $\mu$ and $\nu$.

\section{Lemma 3.2.}

1) $\sum_{\nu \vdash n} c_{\mu \nu}^{\lambda}(b)=\sum_{\nu \vdash n} c_{\nu \mu}^{\lambda}(b)=\left|\mathcal{C}_{\mu}\right|(1+b)^{n-l(\mu)}, \quad$ for $\lambda, \mu \vdash n$,

2) $\sum_{\mu, \nu \vdash n} c_{\mu \nu}^{\lambda}(b)=(n+(n-1) b)(n-1+(n-2) b) \cdots(3+2 b)(2+b), \quad$ for $\lambda \vdash n$.

Proof. 1) Under the substitution $\mathbf{z}=(1,0, \ldots)=\mathbf{e}_{1}$, we have $p_{\nu}\left(\mathbf{e}_{1}\right)=1$ for all $\nu$, from (8). Thus from (16)

$$
\sum_{\nu \vdash n} c_{\mu \nu}^{\lambda}(b)=\left[t^{n} \frac{p_{\lambda}(\mathbf{x})\left|\mathcal{C}_{\lambda}\right|}{n !(1+b)^{l(\lambda)}} p_{\mu}(\mathbf{y})\right] \Phi\left(\mathbf{x}, \mathbf{y}, \mathbf{e}_{1} ; t, 1+b\right) .
$$


But $J_{\theta}\left(\mathbf{e}_{1} ; 1+b\right)=0$ for $l(\theta)>1$ from (8) so by (1)

$$
\begin{gathered}
\Phi\left(\mathbf{x}, \mathbf{y}, \mathbf{e}_{1} ; t, 1+b\right)=\sum_{n \geq 0} \frac{t^{n}}{\left\langle J_{[n]}, J_{[n]}\right\rangle_{1+b}} J_{[n]}(\mathbf{x} ; 1+b) J_{[n]}(\mathbf{y} ; 1+b) J_{[n]}\left(\mathbf{e}_{1} ; 1+b\right) \\
=\sum_{n \geq 0} t^{n} \sum_{\lambda, \mu \vdash n}\left|\mathcal{C}_{\lambda}\right|(1+b)^{n-l(\lambda)} p_{\lambda}(\mathbf{x})\left|\mathcal{C}_{\mu}\right|(1+b)^{n-l(\mu)} p_{\mu}(\mathbf{y}) \frac{1}{n !(1+b)^{n}},
\end{gathered}
$$

from (8) and (9), since

$$
\frac{J_{[n]}\left(\mathbf{e}_{1} ; 1+b\right)}{\left\langle J_{[n]}, J_{[n]}\right\rangle_{1+b}}=\frac{(2+b)(3+2 b) \cdots(n+(n-1) b)}{n !(1+b)^{n}(2+b)(3+2 b) \cdots(n+(n-1) b)} .
$$

The result follows.

2) This follows by summing result (1) above over $\mu \vdash n$, using the cycle index polynomial, or considering the two specializations $\mathbf{y}=\mathbf{e}_{1}, \mathbf{z}=\mathbf{e}_{1}$ and proceeding as in the proof of (1).

b) The case $\lambda=\left[1^{n}\right]$. We can explicitly evaluate $c_{\mu \nu}^{\lambda}(b)$ in this case in a compact form.

Lemma 3.3. For $\mu, \nu \vdash n$,

$$
c_{\mu \nu}^{\left[1^{n}\right]}(b)= \begin{cases}\left|\mathcal{C}_{\mu}\right|(1+b)^{n-l(\mu)}, & \mu=\nu \\ 0, & \mu \neq \nu\end{cases}
$$

Proof. From (16),

$$
\begin{aligned}
c_{\mu \nu}^{\left[1^{n}\right]}(b) & =\left[t^{n} \frac{p_{1}^{n}(\mathbf{x})}{n !} \frac{p_{\mu}(\mathbf{y}) p_{\nu}(\mathbf{z})}{(1+b)^{n}}\right] \Phi(\mathbf{x}, \mathbf{y}, \mathbf{z} ; t, 1+b) \\
& =\left[t^{n} \frac{p_{\mu}(\mathbf{y}) p_{\nu}(\mathbf{z})}{n !(1+b)^{n}}\right] \sum_{\theta \in \mathcal{P}} \frac{1}{\left\langle J_{\theta}, J_{\theta}\right\rangle_{1+b}} J_{\theta}(\mathbf{y} ; 1+b) J_{\theta}(\mathbf{z} ; 1+b) t^{|\theta|}
\end{aligned}
$$

since $\left[p_{1}^{n}\right] J_{\theta}=1$ for all $\theta \vdash n$, from the property (III) of the Jack symmetric functions (6). But from the Cauchy Identity (10),

(17)

$$
\begin{aligned}
\sum_{\theta \in \mathcal{P}} \frac{1}{\left\langle J_{\theta}, J_{\theta}\right\rangle_{1+b}} J_{\theta}(\mathbf{y} ; 1+b) J_{\theta}(\mathbf{z} ; 1+b) t^{|\theta|} & =\prod_{i, j \geq 1}\left(1-y_{i} z_{j} t\right)^{-1 /(1+b)} \\
& =\exp \left\{\frac{1}{1+b} \sum_{k \geq 1} \frac{t^{k}}{k} p_{k}(\mathbf{y}) p_{k}(\mathbf{z})\right\} \\
& =1+\sum_{n \geq 1} \sum_{\gamma \vdash n} \frac{t^{n}\left|\mathcal{C}_{\gamma}\right|}{n !(1+b)^{l(\gamma)}} p_{\gamma}(\mathbf{y}) p_{\gamma}(\mathbf{z})
\end{aligned}
$$

and the result follows.

c) The case $\lambda=\left[21^{n-2}\right]$. In this case we give a generating series. The result is more complicated, but will be needed in Section 4 in the discussion of a conjectured combinatorial interpretation for $c_{\mu \nu}^{\lambda}(b)$.

\section{Lemma 3.4.}

$$
\sum_{n \geq 2} \frac{t^{n-2}}{(n-2) !} \sum_{\mu, \nu \vdash n} c_{\mu \nu}^{\left[21^{n-2}\right]}(b) p_{\mu}(\mathbf{y}) p_{\nu}(\mathbf{z})=\left(\exp \sum_{j \geq 1}(1+b)^{j-1} \frac{t^{j}}{j} p_{j}(\mathbf{y}) p_{j}(\mathbf{z})\right) \times
$$




$$
\begin{array}{r}
\times \sum_{k \geq 2}(1+b)^{k-2} t^{k-2}\left\{b(k-1) p_{k}(\mathbf{y}) p_{k}(\mathbf{z})+\sum_{i=1}^{k-1}\left(p_{k}(\mathbf{y}) p_{i}(\mathbf{z}) p_{k-i}(\mathbf{z})\right.\right. \\
\left.\left.+p_{k}(\mathbf{z}) p_{i}(\mathbf{y}) p_{k-i}(\mathbf{y})\right)\right\}
\end{array}
$$

Proof. The differential operator

$$
\widehat{D}\left(\mathbf{y}_{m} ; \alpha\right)=\frac{\alpha}{2} \sum_{i=1}^{m} y_{i}^{2} \frac{\partial^{2}}{\partial y_{i}^{2}}+\sum_{i=1}^{m} \sum_{j \neq i} \frac{y_{i} y_{j}}{y_{i}-y_{j}} \frac{\partial}{\partial y_{i}}
$$

can easily be expressed in the form

$$
\widehat{D}\left(\mathbf{y}_{m} ; \alpha\right)=D\left(\mathbf{y}_{m} ; \alpha\right)-(m-1) \sum_{i=1}^{m} y_{i} \frac{\partial}{\partial y_{i}},
$$

where $D\left(\mathbf{y}_{m} ; \alpha\right)$ is the differential operator given by $(13)$. But $J_{\theta}\left(\mathbf{y}_{m} ; \alpha\right)$ is a homogeneous polynomial of total degree $|\theta|$ in $\mathbf{y}_{m}$, so

$$
\sum_{i=1}^{m} y_{i} \frac{\partial}{\partial y_{i}} J_{\theta}\left(\mathbf{y}_{m} ; \alpha\right)=|\theta| J_{\theta}\left(\mathbf{y}_{m} ; \alpha\right)
$$

and from (11), (12) and (19),

$$
\widehat{D}\left(\mathbf{y}_{m} ; \alpha\right) J_{\theta}\left(\mathbf{y}_{m} ; \alpha\right)=\left(\left[p_{2} p_{1}^{|\theta|-2}\right] J_{\theta}\right) J_{\theta}\left(\mathbf{y}_{m} ; \alpha\right),
$$

for each $m \geq 1$. Now let

$$
F=\frac{1}{2} \sum_{n \geq 2} \frac{t^{n}}{(n-2) !} \frac{1}{(1+b)^{n-1}} \sum_{\mu, \nu \vdash n} c_{\mu \nu}^{\left[21^{n-2}\right]}(b) p_{\mu}(\mathbf{y}) p_{\nu}(\mathbf{z}) .
$$

Applying (20) to (1) and (16) gives, for each $m \geq 1$,

$$
F=\widehat{D}\left(\mathbf{y}_{m} ; \alpha\right) G
$$

where

$$
\begin{aligned}
G & =\sum_{\theta \in \mathcal{P}} \frac{1}{\left\langle J_{\theta}, J_{\theta}\right\rangle_{1+b}} J_{\theta}\left(\mathbf{y}_{m} ; 1+b\right) J_{\theta}(\mathbf{z} ; 1+b) t^{|\theta|} \\
& =\prod_{i=1}^{m} \prod_{j \geq 1}\left(1-y_{i} z_{j}\right)^{-1 /(1+b)}
\end{aligned}
$$

from the Cauchy Identity (10). Thus, from (17)

$$
\frac{\partial}{\partial y_{i}} G=\frac{G}{1+b} \sum_{k \geq 1} t^{k} y_{i}^{k-1} p_{k}(\mathbf{z})
$$

and differentiating further yields

(23)

$$
y_{i}^{2} \frac{\partial^{2}}{\partial y_{i}^{2}} G=\frac{G}{1+b}\left\{\sum_{k \geq 2} t^{k}(k-1) y_{i}^{k} p_{k}(\mathbf{z})+\frac{1}{1+b}\left(\sum_{k \geq 1} t^{k} y_{i}^{k} p_{k}(\mathbf{z})\right)^{2}\right\}
$$


and for $i \neq j$,

$$
\frac{y_{i} y_{j}}{y_{i}-y_{j}}\left(\frac{\partial}{\partial y_{i}}-\frac{\partial}{\partial y_{j}}\right) G=\frac{G}{1+b} \sum_{k \geq 2} t^{k} p_{k}(\mathbf{z}) \sum_{l=1}^{k-1} y_{i}^{l} y_{j}^{k-l}
$$

Now combining (23) and (24) with (18) and (22) gives, after routine manipulation,

$$
\begin{aligned}
F=\frac{G}{2(1+b)} \sum_{k \geq 2} t^{k}\{b( & -1) p_{k}\left(\mathbf{y}_{m}\right) p_{k}(\mathbf{z}) \\
& \left.+\sum_{i=1}^{k-1}\left(p_{k}\left(\mathbf{y}_{m}\right) p_{i}(\mathbf{z}) p_{k-i}(\mathbf{z})+p_{k}(\mathbf{z}) p_{i}\left(\mathbf{y}_{m}\right) p_{k-i}\left(\mathbf{y}_{m}\right)\right)\right\} .
\end{aligned}
$$

The result follows by substituting for $F$ and $G$ in this equation via (17) and (21), then replacing $t$ by $t(1+b), \mathbf{y}_{m}$ by $\mathbf{y}$, and multiplying on both sides by $2(1+b)^{-1} t^{-2}$.

3.3. A positivity conjecture. The marginal sums for $c_{\mu \nu}^{\lambda}(b)$ evaluated in Lemma 3.2 are nonnegative integer polynomials in $b$ and, by Lemmas 3.3 and 3.4, $c_{\mu \nu}^{\lambda}(b)$ itself is a polynomial in $b$ with nonnegative integer coefficients when $\lambda=\left[1^{n}\right]$ or $\left[21^{n-2}\right]$. Table 1 , which gives the nonzero $c_{\mu \nu}^{\lambda}(b)$ for $\mu \geq \nu,\left(\operatorname{since} c_{\mu \nu}^{\lambda}(b)=c_{\nu \mu}^{\lambda}(b)\right)$ shows that this is also true of them individually for all $\lambda, \mu, \nu \vdash n \leq 4$. In fact, using the SF package [19], we have computed $c_{\mu \nu}^{\lambda}(b)$ for all $\lambda, \mu, \nu \vdash n \leq 8$, and found in all cases that $c_{\mu \nu}^{\lambda}(b)$ is a nonnegative integer polynomial in $b$. We conjecture that this is always the case.

Conjecture 3.5. For all $\lambda, \mu, \nu \vdash n \geq 1$,

$$
c_{\mu \nu}^{\lambda}(b) \text { is a nonnegative integer polynomial in } b .
$$

Note that if we express the connection series in terms of $\alpha$ then we can have negative coefficients in general; for example,

$$
c_{\mu \nu}^{\lambda}(\alpha-1)=2 \alpha^{2}-3 \alpha+2
$$

when $\lambda=\mu=\nu=[3]$. This is why the Jack parameter has been shifted by the substitution $\alpha=b+1$.

Under the assumption that Conjecture 3.5 is true, information is now obtained about the degree of the polynomial $c_{\mu \nu}^{\lambda}(b)$ in $b$.

Proposition 3.6. If Conjecture 3.5 holds, then for $\lambda, \mu, \nu \vdash n \geq 1$,

1) The degree of $c_{\mu \nu}^{\lambda}(b)$ is at most $n-\min \{l(\mu), l(\nu)\}$.

2) For fixed $\lambda$, the largest power of $b$ appearing among the $c_{\mu \nu}^{\lambda}(b)$ is $n-1$, which uniquely appears in $c_{[n],[n]}^{\lambda}(b)$, with coefficient $(n-1)$ !

Proof. 1) From part (2) of Lemma 3.2, we have

$$
\sum_{\nu \vdash n} c_{\mu \nu}^{\lambda}(b)=\left|\mathcal{C}_{\mu}\right|(1+b)^{n-l(\mu)}
$$

and

$$
\sum_{\mu \vdash n} c_{\mu \nu}^{\lambda}(b)=\left|\mathcal{C}_{\nu}\right|(1+b)^{n-l(\nu)} .
$$

But the degree of $(1+b)^{n-l(\mu)}$ is $n-l(\mu)$. We assume that Conjecture 3.5 is true. Then the degree of $c_{\mu \nu}^{\lambda}(b)$ exceeding $n-l(\mu)$ would contradict (25), and the degree of $c_{\mu \nu}^{\lambda}(b)$ exceeding $n-l(\nu)$ would contradict (26), so the result follows. 
TABLE 1. The connection series $c_{\mu \nu}^{\lambda}(b)$ for $\lambda, \mu, \nu \vdash n \leq 4$, and $\mu \geq \nu$

\begin{tabular}{|c|c|c|c|c|c|c|c|}
\hline$\lambda$ & $\mu$ & $\nu$ & $c_{\mu \nu}^{\lambda}(b)$ & $\lambda$ & $\mu$ & $\nu$ & $c_{\mu \nu}^{\lambda}(b)$ \\
\hline$[1]$ & [1] & {$[1]$} & 1 & \multirow[t]{4}{*}[\begin{array}{ll}{3}&{1}\end{array}]{} & \multirow[t]{4}{*}[4]{} & {$[4]$} & $6 b^{3}+9 b^{2}+6 b+3$ \\
\hline \multirow[t]{2}{*}[2]{} & {$[2]$} & {$[2]$} & $b$ & & & {$\left[\begin{array}{ll}3 & 1\end{array}\right]$} & $6 b^{2}+6 b$ \\
\hline & & {$\left[1^{2}\right]$} & 1 & & & {$\left[2^{2}\right]$} & $3 b^{2}+3 b$ \\
\hline \multirow[t]{2}{*}[1^{2}]{} & {$[2]$} & {$[2]$} & $b+1$ & & & {$\left[21^{2}\right]$} & $3 b+3$ \\
\hline & {$\left[1^{2}\right]$} & {$\left[1^{2}\right]$} & 1 & \multirow[t]{4}{*}[\begin{array}{ll}{3}&{1}\end{array}]{} & \multirow[t]{4}{*}[\begin{array}{ll}{3}&{1}\end{array}]{} & {$[31]^{3}$} & $2 b^{2}+4 b+4$ \\
\hline \multirow[t]{6}{*}[3]{} & {$[3]$} & {$[3]$} & $2 b^{2}+b+1$ & & & {$\left[2^{2}\right]$} & $3 b+3$ \\
\hline & & {$\left[\begin{array}{ll}2 & 1\end{array}\right]$} & $3 b$ & & & {$\left[21^{2}\right]$} & $3 b$ \\
\hline & & {$\left[1^{3}\right]$} & 1 & & & {$\left[1^{4}\right]$} & 1 \\
\hline & {$\left[\begin{array}{ll}2 & 1\end{array}\right]$} & {$[3]$} & $3 b$ & \multirow{10}{*}[2^{2}]{} & {$\left[21^{2}\right]$} & {$\left[21^{2}\right]$} & 3 \\
\hline & & {$\left[\begin{array}{ll}2 & 1\end{array}\right]$} & 3 & & {$[4]$} & {$[4]$} & $6 b^{3}+8 b^{2}+4 b+2$ \\
\hline & {$\left[1^{3}\right]$} & {$[3]$} & 1 & & & {$\left[\begin{array}{ll}3 & 1\end{array}\right]$} & $8 b^{2}+8 b$ \\
\hline \multirow[t]{4}{*}[\begin{array}{ll}{2}&{1}\end{array}]{} & {$[3]$} & {$[3]$} & $2 b^{2}+2 b$ & & & {$\left[2^{2}\right]$} & $2 b^{2}+2 b$ \\
\hline & & {$\left[\begin{array}{ll}2 & 1\end{array}\right]$} & $2 b+2$ & & & {$\left[21^{2}\right]$} & $4 b+4$ \\
\hline & {$\left[\begin{array}{ll}2 & 1\end{array}\right]$} & {$\left[\begin{array}{ll}2 & 1\end{array}\right]$} & $b$ & & {$\left[\begin{array}{ll}3 & 1\end{array}\right]$} & {$[31]$} & $8 b+8$ \\
\hline & & {$\left[1^{3}\right]$} & 1 & & {$\left[2^{2}\right]$} & {$\left[2^{2}\right]$} & $b^{2}+2 b+2$ \\
\hline \multirow[t]{3}{*}[1^{3}]{} & {$[3]$} & {$[3]$} & $2 b^{2}+4 b+2$ & & & {$\left[21^{2}\right]$} & $2 b$ \\
\hline & {$\left[\begin{array}{ll}2 & 1\end{array}\right]$} & {$[21]$} & $3 b+3$ & & & {$\left[1^{4}\right]$} & 1 \\
\hline & {$\left[1^{3}\right]$} & {$\left[1^{3}\right]$} & 1 & & {$\left[21^{2}\right]$} & {$\left[21^{2}\right]$} & 2 \\
\hline \multirow[t]{11}{*}[4]{} & {$[4]$} & {$[4]$} & $6 b^{3}+7 b^{2}+7 b$ & \multirow[t]{9}{*}[21^{2}]{} & {$[4]$} & {$[4]$} & $6 b^{3}+12 b^{2}+6 b$ \\
\hline & & {$\left[\begin{array}{ll}3 & 1\end{array}\right]$} & $8 b^{2}+4 b+4$ & & & {$\left[\begin{array}{ll}3 & 1\end{array}\right]$} & $4 b^{2}+8 b+4$ \\
\hline & & {$\left[2^{2}\right]$} & $3 b^{2}+b+1$ & & & {$\left[2^{2}\right]$} & $2 b^{2}+4 b+2$ \\
\hline & & {$\left[21^{2}\right]$} & $6 b$ & & {$[31]$} & {$[31]$} & $4 b^{2}+4 b$ \\
\hline & & {$\left[1^{4}\right]$} & 1 & & & {$\left[21^{2}\right]$} & $4 b+4$ \\
\hline & {$[31]$} & {$\left[\begin{array}{ll}3 & 1\end{array}\right]$} & $8 b$ & & {$\left[2^{2}\right]$} & {$\left[2^{2}\right]$} & $b^{2}+b$ \\
\hline & & {$\left[2^{2}\right]$} & $4 b$ & & & {$\left[21^{2}\right]$} & $b+1$ \\
\hline & & {$\left[\begin{array}{lll}2 & 1^{2}\end{array}\right]$} & 4 & & {$\left[21^{2}\right]$} & {$\left[21^{2}\right]$} & $b$ \\
\hline & {$\left[2^{2}\right]$} & {$\left[2^{2}\right]$} & $b$ & & & {$\left[1^{4}\right]$} & 1 \\
\hline & & 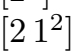 & 2 & \multirow[t]{2}{*}[1^{4}]{} & {$[4]$} & {$[4]$} & $6 b^{3}+18 b^{2}+18 b+6$ \\
\hline & & & & & $\begin{array}{l}{[31]} \\
{\left[2^{2}\right]} \\
{\left[\begin{array}{ll}2 & 1^{2} \\
{\left[1^{4}\right]}\end{array}\right.}\end{array}$ & $\begin{array}{l}{[31]} \\
{\left[2^{2}\right]} \\
{\left[\begin{array}{ll}2 & 1^{2} \\
{\left[1^{4}\right]}\end{array}\right.}\end{array}$ & $\begin{array}{c}8 b^{2}+16 b+8 \\
3 b^{2}+6 b+3 \\
6 b+6 \\
1\end{array}$ \\
\hline
\end{tabular}

2) Part (1) of the result gives immediately that the degree of $c_{\mu \nu}^{\lambda}(b)$ is at most $n-1$, and is strictly less than $n-1$ unless $\mu=\nu=[n]$. But from (25) with $\mu=[n]$ we get

$$
\sum_{\nu \vdash n} c_{[n], \nu}^{\lambda}(b)=(n-1) !(1+b)^{n-1} .
$$

Thus the only contribution to the term $(n-1) ! b^{n-1}$ on the right-hand side of this sum comes from $c_{[n],[n]}^{\lambda}(b)$, and the result follows. 
The above result for terms of highest degree can be verified for $n \leq 4$ in Table 1 . For terms of lowest degree we have been able to deduce less information. Of course, the constant terms are the connection coefficients in the symmetric group, from Proposition 3.1 (1). For more information about when these are 0 and some extreme non-zero values see [7].

We note in passing a curiosity of the data in Table 1, that all entries are unimodal, and we further conjecture that, for all $\lambda, \mu, \nu \vdash n \geq 1, c_{\mu \nu}^{\lambda}(b)$ is unimodal.

\section{The Matchings-Jack Conjecture}

Let $\mathcal{F}_{\mathcal{S}}$ be the set of all matchings on a set $\mathcal{S}$ of even cardinality. For $\delta_{1}, \delta_{2}, \ldots \in$ $\mathcal{F}_{\mathcal{S}}$, let $G\left(\delta_{1}, \delta_{2}, \ldots\right)$ be the multi-graph with vertex-set $\mathcal{S}$ whose edges are formed by the pairs in $\delta_{1}, \delta_{2}, \ldots$. Now $G\left(\delta_{1}, \delta_{2}\right)$ consists of disjoint even cycles, since each vertex has degree 2 , and around each cycle the edges alternate between $\delta_{1}$ and $\delta_{2}$, so we let $\Lambda\left(\delta_{1}, \delta_{2}\right)$ be the partition specifying half the lengths of the cycles in $G\left(\delta_{1}, \delta_{2}\right)$.

Let $\mathcal{F}_{n}$ be the set of matchings on $\mathcal{N}_{n, \widehat{n}}=\{1, \widehat{1}, \ldots, n, \widehat{n}\}$. For convenience, the sets $\{1, \ldots, n\}$ and $\{\widehat{1}, \ldots, \widehat{n}\}$ will be called the classes of $\mathcal{N}_{n, \widehat{n}}$. Thus pairs in a matching contain either two elements of the same class (we call these withinclass pairs) or elements of different classes (we call these between-class pairs). A matching $\delta$ in which every pair is a between-class pair is called a bipartite matching, since in this case $G(\delta)$ is a subgraph of the complete bipartite graph on vertex-sets $\{1, \ldots, n\}$ and $\{\widehat{1}, \ldots, \widehat{n}\}$.

Now we introduce some specific bipartite matchings in $\mathcal{F}_{n}$. First, let

$$
\varepsilon=\{\{1, \widehat{1}\}, \ldots,\{n, \widehat{n}\}\} .
$$

Then, for each $\lambda \vdash n$, let

$$
\begin{aligned}
\delta_{\lambda}= & \{1, \widehat{2}\},\{2, \widehat{3}\}, \ldots,\left\{\lambda_{1}-1, \widehat{\lambda_{1}}\right\},\left\{\lambda_{1}, \widehat{1}\right\}, \\
& \left.\left\{\lambda_{1}+1, \widehat{\lambda_{1}+2}\right\}, \ldots,\left\{\lambda_{1}+\lambda_{2}-1, \widehat{\lambda_{1}+\lambda_{2}}\right\},\left\{\lambda_{1}+\lambda_{2}, \widehat{\lambda_{1}+1}\right\}, \ldots\right\} .
\end{aligned}
$$

In particular, $\delta_{\left[1^{n}\right]}=\varepsilon$. Note that $\varepsilon$ and $\delta_{\lambda}$ are bipartite matchings, and that $\Lambda\left(\varepsilon, \delta_{\lambda}\right)=\lambda$ for all $\lambda \vdash n$.

There is a close relationship between connection coefficients in the double coset algebra and matchings, since (see [10], Lemma 3.2)

$$
\frac{b_{\mu \nu}^{\lambda}}{\left|\mathcal{B}_{n}\right|}=\left|\left\{\delta \in \mathcal{F}_{n}: \Lambda(\delta, \varepsilon)=\mu, \Lambda\left(\delta, \delta_{\lambda}\right)=\nu\right\}\right| .
$$

The specializations of $c_{\mu \nu}^{\lambda}(b)$ given in Proposition 3.1 for $b=0,1$ involve connection coefficients in both the double coset algebra and the conjugacy class algebra. These specializations are made more uniform by the following combinatorial restatements in terms of matchings.

Proposition 4.1. For $\lambda, \mu, \nu \vdash n$,

1) $c_{\mu \nu}^{\lambda}(0)=\mid\left\{\delta \in \mathcal{F}_{n}: \Lambda(\delta, \varepsilon)=\mu, \Lambda\left(\delta, \delta_{\lambda}\right)=\nu, \delta\right.$ is bipartite $\} \mid$,

2) $c_{\mu \nu}^{\lambda}(1)=\left|\left\{\delta \in \mathcal{F}_{n}: \Lambda(\delta, \varepsilon)=\mu, \Lambda\left(\delta, \delta_{\lambda}\right)=\nu\right\}\right|$.

Proof. 1) Let $\tau=\left(12 \cdots \lambda_{1}\right)\left(\lambda_{1}+1 \cdots \lambda_{1}+\lambda_{2}\right) \cdots \in \mathcal{C}_{\lambda}$. Then

$$
a_{\mu \nu}^{\lambda}=\left[\mathrm{C}_{\lambda}\right] \mathrm{C}_{\mu} \mathrm{C}_{\nu}=\left|\left\{\sigma \in \mathcal{C}_{\mu}, \rho \in \mathcal{C}_{\nu}: \sigma \rho=\tau\right\}\right|=\left|\left\{\sigma \in \mathcal{C}_{\mu}: \sigma^{-1} \tau \in \mathcal{C}_{\nu}\right\}\right| .
$$


For $\sigma \in \mathcal{C}_{\mu}$ we define $\delta \in \mathcal{F}_{n}$ by

$$
\delta=\{(\widehat{1}, \sigma(1)),(\widehat{2}, \sigma(2)), \ldots,(\widehat{n}, \sigma(n))\} .
$$

Then $\delta$ is a bipartite matching such that $\Lambda(\delta, \varepsilon)=\mu$, and $\Lambda\left(\delta, \delta_{\lambda}\right)=\nu$ if and only if $\sigma^{-1} \tau \in \mathcal{C}_{\nu}$. Moreover, this is reversible, since every bipartite $\delta \in \mathcal{F}_{n}$ uniquely determines $\sigma \in \mathfrak{S}_{n}$ with the required properties. The result follows from Proposition 3.1 .

2) This result has been given as (27).

Under the assumption that Conjecture 3.5 is true, the combinatorial form of the specializations to $b=0$ and 1 in Proposition 4.1 above suggest the following stronger conjecture, that $c_{\mu \nu}^{\lambda}(b)$ is a counting series for matchings.

Conjecture 4.2 (Matchings-Jack Conjecture). For $\lambda, \mu, \nu \vdash n$,

$$
c_{\mu \nu}^{\lambda}(b)=\sum_{\substack{\delta \in \mathcal{F}_{n} \\ \Lambda(\delta, \varepsilon)=\mu, \Lambda\left(\delta, \delta_{\lambda}\right)=\nu}} b^{\mathrm{wt}_{\lambda}(\delta)}
$$

for nonnegative integer $\mathrm{wt}_{\lambda}(\delta)$, where $\mathrm{wt}_{\lambda}(\delta)=0$ if and only if $\delta$ is bipartite, and $\mathrm{wt}_{\lambda}(\delta) \leq n-\min \{l(\mu), l(\nu)\}$.

In the context of this conjecture, the shifted Jack parameter $b$ is a combinatorial marker for a family of statistics of non-bipartiteness for matchings, indexed by $\lambda$. In the next section, we describe some combinatorial statistics for matchings that serve as appropriate choices of $\mathrm{wt}_{\lambda}$ for $\lambda=\left[1^{n}\right]$ and $\lambda=\left[21^{n-2}\right]$, thus achieving partial success in establishing the Matchings-Jack Conjecture by proving it in these cases.

\section{Combinatorial Statistics FOR Matchings}

For a matching $\delta \in \mathcal{F}_{n}$, it will be convenient to describe the pairs in $\delta$ relative to their appearance in the cycles of $G(\delta, \varepsilon)$, as follows. For a given cycle of $G(\delta, \varepsilon)$, let $j$ be the smallest value such that $\{j, \widehat{j}\} \in \varepsilon$ is in the cycle. Now traverse the cycle beginning with the pair of $\delta$ containing $j$, and terminating with the pair of $\delta$ containing $\widehat{j}$ (these are the same when the cycle has length two). This orders the pairs of $\delta$ in the cycle, as well as directing them; it also orders and directs the pairs of $\varepsilon$ in the cycle, with the convention that we begin at the ordered pair $(\widehat{j}, j)$. Moreover, the disjoint union of the pairs of $\delta$ on a cycle is the same as the disjoint union of the pairs of $\varepsilon$ on the cycle. Thus, if we know the order and directions of the pairs of $\delta$ on a cycle then we know the order and directions of the pairs of $\varepsilon$ on the cycle, and vice-versa.

Example 5.1. For $n=5$ consider the matching

$$
\gamma=\{\{1, \widehat{4}\},\{\widehat{1}, \widehat{2}\},\{2,4\},\{3,5\},\{\widehat{3}, \widehat{5}\}\} .
$$

Then $G(\gamma, \varepsilon)$ has two cycles, one with vertex-set $\{1, \widehat{1}, 2, \widehat{2}, 4, \widehat{4}\}$ and the other with vertex-set $\{3, \widehat{3}, 5, \widehat{5}\}$. Traversing the first of these as described above gives the pairs of $\gamma$ in the order $(1, \widehat{4}),(4,2),(\widehat{2}, \widehat{1})$, with directions as indicated by the ordered pairs; the pairs of $\varepsilon$ appear in order $(\widehat{1}, 1),(\widehat{4}, 4),(2, \widehat{2})$, again with directions as indicated. Traversing the second cycle gives the pairs of $\gamma$ ordered and directed as $(3,5),(\widehat{5}, \widehat{3})$, and the pairs of $\varepsilon$ as $(\widehat{3}, 3),(5, \widehat{5})$. 
From this point of view the following relationship between permutations and matchings is straightforward.

Proposition 5.2. For $\mu \vdash n$,

$$
\left|\left\{\delta \in \mathcal{F}_{n}: \Lambda(\delta, \varepsilon)=\mu\right\}\right|=\left|\mathcal{C}_{\mu}\right| 2^{n-l(\mu)} .
$$

Proof. For each directed cycle $\left(i_{1} i_{2} \ldots i_{k}\right)$, of length $k \geq 1$, in the disjoint cycle representation of a permutation $\sigma \in \mathfrak{S}_{n}$ we create $2^{k-1}$ choices for a cycle, of length $2 k$, in $G(\delta, \varepsilon)$ for $\delta \in \mathcal{F}_{n}$, as follows. Let the order of the pairs of $\varepsilon$ in the cycle of $G(\delta, \varepsilon)$ be $\left\{i_{1}, \widehat{i}_{1}\right\},\left\{i_{2}, \widehat{i}_{2}\right\}, \ldots,\left\{i_{k}, \widehat{i}_{k}\right\}$. Now the first pair $\left\{i_{1}, \widehat{i}_{1}\right\}$ is directed as $\left(\hat{i}_{1}, i_{1}\right)$ by convention. The remaining $k-1$ pairs can be directed independently in either of the two ways $\left(i_{m}, \widehat{i}_{m}\right)$ or $\left(\widehat{i}_{m}, i_{m}\right)$, for $m=2, \ldots, k$. But this uniquely determines the positions of the pairs of $\delta$ on the cycle. Thus, all $\delta \in \mathcal{F}_{n}$ with $\Lambda(\delta, \varepsilon)=\mu$ are created exactly once by carrying this out for all the cycles of $\sigma \in \mathcal{C}_{\mu}$, and the result follows.

5.1. The first statistic. The first statistic for matchings, defined below as stat ${ }_{1}$, will account for the case $\lambda=\left[1^{n}\right]$ of the Matchings-Jack Conjecture.

Definition 5.3. For $\delta \in \mathcal{F}_{n}$, $\operatorname{stat}_{1}(\delta)$ is the number of within-class pairs of $\delta$ that do not terminate their cycles in $G(\delta, \varepsilon)$.

A convenient way of thinking about such a statistic is to regard each pair of $\delta$ as "contributing" or "not contributing" to the statistic. Thus, for stat 1 , a pair of $\delta$ contributes if it is a within-class pair and it is not the terminating edge (in either direction) in its cycle of $G(\delta, \varepsilon)$, and does not contribute otherwise.

Example 5.4. For the matching $\gamma$ given in Example 5.1 we calculate that $\operatorname{stat}_{1}(\gamma)$ $=2$. In this case, the pairs that contribute to the statistic are $\{2,4\}$ and $\{3,5\}$. The pairs $\{\widehat{1}, \widehat{2}\}$ and $\{\widehat{3}, \widehat{5}\}$ do not contribute since, although they are within-class pairs, they are terminal in their respective cycles.

5.2. Proof of the Matchings-Jack Conjecture for $\lambda=\left[1^{n}\right]$. The counting series for matchings with respect to the statistic stat ${ }_{1}$ is readily determined.

Proposition 5.5. For $\mu \vdash n$,

$$
\sum_{\substack{\delta \in \mathcal{F}_{n} \\ \Lambda(\delta, \varepsilon)=\mu}} b^{\text {stat }_{1}(\delta)}=\left|\mathcal{C}_{\mu}\right|(1+b)^{n-l(\mu)} .
$$

Proof. In the proof of Proposition 5.2, note that the choices of direction for each edge of $\varepsilon$ determines precisely whether the immediately preceding edge of $\delta$ joins two elements of the same class or elements of different classes. The terminal edge of $\delta$ in the cycle has no such choice because of the convention for the initial edge of $\varepsilon$. Thus a cycle of length $k$ contributes a factor of $(1+b)^{k-1}$ to the generating function for stat $_{1}$, so an element of $\mathcal{C}_{\mu}$ contributes $(1+b)^{n-l(\mu)}$. The result follows since there are $\left|\mathcal{C}_{\mu}\right|$ elements of $\mathcal{C}_{\mu}$.

We can immediately deduce that this provides a suitable choice for the conjectured statistic $\mathrm{wt}_{\lambda}$ in the case $\lambda=\left[1^{n}\right]$.

Corollary 5.6. The Matchings-Jack Conjecture (4.2) is true for $\lambda=\left[1^{n}\right]$. 
Proof. When $\lambda=\left[1^{n}\right]$, we have $\delta_{\lambda}=\varepsilon$, so in this case

$$
\sum_{\substack{\delta \in \mathcal{F}_{n} \\ \Lambda(\delta, \varepsilon)=\mu, \Lambda\left(\delta, \delta_{\lambda}\right)=\nu}} b^{\text {stat }_{1}(\delta)}=\sum_{\substack{\delta \in \mathcal{F}_{n} \\ \Lambda(\delta, \varepsilon)=\mu}} b^{\text {stat }_{1}(\delta)}
$$

for $\mu=\nu$, and is 0 otherwise. Thus from Proposition 5.5 and Lemma 3.3, stat st $_{1}$ is suitable choice for $\mathrm{wt}_{\lambda}$ when $\lambda=\left[1^{n}\right]$, establishing the Conjecture in this case.

5.3. The second statistic. The second statistic for matchings is a modification of stat $_{1}$, and will account for the case $\lambda=\left[21^{n-2}\right]$ of the Matchings-Jack Conjecture.

Definition 5.7. For $\delta \in \mathcal{F}_{n}$, $\operatorname{stat}_{2}(\delta)$ is defined as follows:

1. If $\{1, \widehat{1}\}$ and $\{2, \widehat{2}\}$ lie on different cycles of $G(\delta, \varepsilon)$ then $\operatorname{stat}_{2}(\delta)$ has the same value as $\operatorname{stat}_{1}(\delta)$.

2. Otherwise,

(a) if 2 appears before $\widehat{2}$ on the cycle containing $\{1, \widehat{1}\}$ and $\{2, \widehat{2}\}$, then

(i) the pair of $\delta$ containing 2 always contributes to $\operatorname{stat}_{2}(\delta)$,

(ii) all other pairs in $\delta$ are treated as in $s t_{1}$;

(b) if $\widehat{2}$ appears before 2 , then

(i) the pair of $\delta$ containing $\widehat{2}$ never contributes to $\operatorname{stat}_{2}(\delta)$,

(ii) all other pairs in $\delta$ are treated as in stat 1 .

Example 5.8. For the matching $\gamma$ given in Example 5.1 we calculate $\operatorname{stat}_{2}(\gamma)=2$. We are in the case in which $\{1, \widehat{1}\}$ and $\{2, \widehat{2}\}$ lie on the same cycle and, on that cycle, 2 appears before $\widehat{2}$, so the pairs that contribute to the statistic are again $\{2,4\}$ and $\{3,5\}$.

Example 5.9. Consider the matching

$$
\eta=\{\{1,4\},\{\widehat{1}, \widehat{5}\},\{2,5\},\{\widehat{2}, \widehat{4}\},\{3, \widehat{3}\}\} .
$$

Then $G(\eta, \varepsilon)$ has two cycles, and traversing these cycles gives the edges of $\eta$ ordered and directed as $(1,4)(\widehat{4}, \widehat{2})(2,5)(\widehat{5}, \widehat{1})$ in one cycle and $(3, \widehat{3})$ in the second cycle. We thus have $\operatorname{stat}_{1}(\eta)=3$, with pairs $\{1,4\},\{2,5\}$ and $\{\widehat{2}, \widehat{4}\}$ contributing. However, we have $\operatorname{stat}_{2}(\eta)=2$, with pairs $\{1,4\}$ and $\{2,5\}$ contributing; the pair $\{\widehat{2}, \widehat{4}\}$ does not contribute even though $\widehat{2}$ and $\widehat{4}$ are in the same class, since we are in the case in which $\{1, \widehat{1}\}$ and $\{2, \widehat{2}\}$ appear on the same cycle of $G(\eta, \varepsilon)$ with $\widehat{2}$ before 2 .

5.4. Proof of the Matchings-Jack Conjecture for $\lambda=\left[21^{n-2}\right]$. We determine

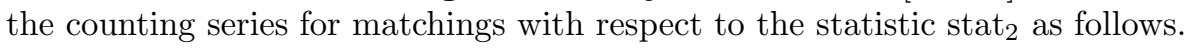

Lemma 5.10. Let $\lambda=\left[21^{n-2}\right]$. For $\mu, \nu \vdash n$,

$$
\begin{gathered}
\sum_{\substack{\delta \in \mathcal{F}_{n} \\
\Lambda(\delta, \varepsilon)=\mu,\left(\delta, \delta_{\lambda}\right)=\nu}} b^{\mathrm{stat}_{2}(\delta)}=\left[\frac{t^{n-2}}{(n-2) !} p_{\mu}(\mathbf{y}) p_{\nu}(\mathbf{z})\right]\left(\exp \sum_{j \geq 1}(1+b)^{j-1} \frac{t^{j}}{j} p_{j}(\mathbf{y}) p_{j}(\mathbf{z})\right) \\
\sum_{k \geq 2}(1+b)^{k-2} t^{k-2}\left\{b(k-1) p_{k}(\mathbf{y}) p_{k}(\mathbf{z})\right. \\
\left.+\sum_{i=1}^{k-1}\left(p_{k}(\mathbf{y}) p_{i}(\mathbf{z}) p_{k-i}(\mathbf{z})+p_{k}(\mathbf{z}) p_{i}(\mathbf{y}) p_{k-i}(\mathbf{y})\right)\right\}
\end{gathered}
$$


Proof. Let $\lambda=\left[21^{n-2}\right]$, so $\delta_{\lambda}=\{\{1, \widehat{2}\},\{\widehat{1}, 2\},\{3, \widehat{3}\}, \ldots,\{n, \widehat{n}\}\}$ for $n \geq 2$, and define the counting series

$$
\Theta(\mathbf{y}, \mathbf{z} ; t, b)=\sum_{n \geq 2} \frac{t^{n-2}}{(n-2) !} \sum_{\delta \in \mathcal{F}_{n}} b^{\text {stat }_{2}(\delta)} p_{\Lambda(\delta, \varepsilon)}(\mathbf{y}) p_{\Lambda\left(\delta, \delta_{\lambda}\right)}(\mathbf{z}) .
$$

Then $\Theta$ has $t$ as an exponential marker for the pairs in $\mathcal{A}=\{\{3, \widehat{3}\}, \ldots,\{n, \widehat{n}\}\}$, $p_{i}(\mathbf{y})$ as a (ordinary) marker for $i$-cycles in $G(\delta, \varepsilon), p_{i}(\mathbf{z})$ as a (ordinary) marker for $i$-cycles in $G\left(\delta, \delta_{\lambda}\right)$, for $i \geq 1$, and b as a (ordinary) marker for pairs in $\delta$ contributing to stat 2 .

We consider separately the three cases for the cycle(s) in $G(\delta, \varepsilon)$ containing $\{1, \widehat{1}\}$ and $\{2, \widehat{2}\}$.

Case 1. Suppose $\{1, \widehat{1}\}$ and $\{2, \widehat{2}\}$ lie on different cycles of $G(\delta, \varepsilon)$ with $m$ elements of $\mathcal{A}$ on the cycle containing $\{1, \widehat{1}\}$ and $j$ elements of $\mathcal{A}$ on the cycle containing $\{2, \widehat{2}\}$, for $m, j \geq 0$. Then the pairs of $\delta$ on these cycles form an $m+j+2$-cycle in $G\left(\delta, \delta_{\lambda}\right)$, so the counting series for these cycles is (see [4] for basic results on the combinatorics associated with exponential and ordinary counting series)

$$
\begin{gathered}
\sum_{m, j \geq 0} m ! \frac{t^{m}}{m !}(1+b)^{m} p_{m+1}(\mathbf{y}) j ! \frac{t^{j}}{j !}(1+b)^{j} p_{j+1}(\mathbf{y}) p_{m+j+2}(\mathbf{z}) \\
=\sum_{k \geq 2} \sum_{i=1}^{k-1}(1+b)^{k-2} t^{k-2} p_{i}(\mathbf{y}) p_{k-i}(\mathbf{y}) p_{k}(\mathbf{z})
\end{gathered}
$$

with $i=m+1, k=m+j+2$.

Case 2. Suppose $\{1, \widehat{1}\}$ and $\{2, \widehat{2}\}$ lie on the same cycle of $G(\delta, \varepsilon)$ with 2 appearing before $\widehat{2}$, and that there are $m$ elements of $\mathcal{A}$ between $(\widehat{1}, 1)$ and $(2, \widehat{2})$, and $j$ elements of $\mathcal{A}$ after $(2, \widehat{2})$, for $m, j \geq 0$. Then the pairs of $\delta$ on this cycle form an $m+j+2$-cycle in $G\left(\delta, \delta_{\lambda}\right)$, so the counting series for this cycle is

$$
\begin{gathered}
b \sum_{m, j \geq 0} m ! \frac{t^{m}}{m !}(1+b)^{m} j ! \frac{t^{j}}{j !}(1+b)^{j} p_{m+j+2}(\mathbf{y}) p_{m+j+2}(\mathbf{z}) \\
=b \sum_{k \geq 2}(k-1)(1+b)^{k-2} t^{k-2} p_{k}(\mathbf{y}) p_{k}(\mathbf{z})
\end{gathered}
$$

with $k=m+j+2$.

Case 3. Finally, suppose $\{1, \widehat{1}\}$ and $\{2, \widehat{2}\}$ lie on the same cycle of $G(\delta, \varepsilon)$ with $\widehat{2}$ appearing before 2 , and that there are $m$ elements of $\mathcal{A}$ between $(\widehat{1}, 1)$ and $(\widehat{2}, 2)$, and $j$ elements of $\mathcal{A}$ after $(\widehat{2}, 2)$, for $m, j \geq 0$. Then the pairs of $\delta$ on this cycle form an $m+1$-cycle and a $j+1$-cycle in $G\left(\delta, \delta_{\lambda}\right)$, so the counting series for this case is

$$
\begin{gathered}
\sum_{m, j \geq 0} m ! \frac{t^{m}}{m !}(1+b)^{m} p_{m+1}(\mathbf{z}) j ! \frac{t^{j}}{j !}(1+b)^{j} p_{j+1}(\mathbf{z}) p_{m+j+2}(\mathbf{y}) \\
=\sum_{k \geq 2} \sum_{i=1}^{k-1}(1+b)^{k-2} t^{k-2} p_{i}(\mathbf{z}) p_{k-i}(\mathbf{z}) p_{k}(\mathbf{y})
\end{gathered}
$$

with $i=m+1, k=m+j+2$. 
Now each other cycle in $G(\delta, \varepsilon)$ consists entirely of $j$ elements of $\mathcal{A}$, for some $j \geq 1$, and thus has counting series

$$
\sum_{j \geq 1}(j-1) ! \frac{t^{j}}{j !}(1+b)^{j-1} p_{j}(\mathbf{y}) p_{j}(\mathbf{z}) .
$$

But we have an unordered collection of such cycles, with counting series

$$
\exp \sum_{j \geq 1}(1+b)^{j-1} \frac{t^{j}}{j} p_{j}(\mathbf{y}) p_{j}(\mathbf{z}) .
$$

Thus $\Theta$ is obtained by multiplying (31) by the sum of (28), (29) and (30). The result follows by applying the coefficient operator $\left[\frac{t^{n-2}}{n-2 !} p_{\mu}(\mathbf{y}) p_{\nu}(\mathbf{z})\right]$ to $\Theta$.

We can immediately deduce that stat $_{2}$ provides a suitable choice for the conjectured statistic $\mathrm{wt}_{\lambda}$ in the case $\lambda=\left[21^{n-2}\right]$.

Corollary 5.11. The Matchings-Jack Conjecture (4.2) is true for $\lambda=\left[21^{n-2}\right]$.

Proof. From Lemmas 5.10 and 3.4, stat 2 is a suitable choice for $\mathrm{wt}_{\lambda}$ when $\lambda=$ $\left[21^{n-2}\right]$, establishing the Conjecture in this case.

5.5. Towards other statistics. The algorithms for stat ${ }_{1}$ and $s t_{2} t_{2}$ are given in Definitions 5.3 and 5.7 as a list of cases, but the means by which they were discovered is not evident from their statement. However, there are general strategies that provided a useful framework in finding them, and makes the relationship with $\lambda$ (here $\left[1^{n}\right]$ and $\left[21^{n-2}\right]$, respectively) more explicit.

In both algorithms we examine the pairs of $\delta$ in order of their appearance in the cycles of $G(\delta, \varepsilon)$, thus implicitly ordering (as well as directing) the pairs of $\varepsilon$. From this point of view, the pairs of $\delta$ are of three types. The pairs of $\delta$ that terminate their cycle in $G(\delta, \varepsilon)$ are of type A. Each remaining pair of $\delta$ corresponds to adding a new pair of $\varepsilon$ to their cycle in $G(\delta, \varepsilon)$, in either of two directions. A pair of $\delta$ is of type B if it corresponds in this way to a pair $P$ of $\varepsilon$ that is examined first in the set of all pairs of $\varepsilon$ that are both in the component of $G\left(\varepsilon, \delta_{\lambda}\right)$ containing $P$ and in the component of $G(\delta, \varepsilon)$ containing $P$. Otherwise, it is of type $\mathrm{C}$.

In both algorithms the following rules are used for pairs in $\delta$ of types $\mathrm{A}$ and $\mathrm{B}$ :

A: These pairs do not contribute,

B: These pairs

- contribute if they are within-class pairs,

- do not contribute if they are between-class pairs.

For $\lambda=\left[1^{n}\right]$ there are no pairs of type $\mathrm{C}$ in any matching, and indeed these two rules are simply a restatement of the algorithm given for this case in Definition 5.3.

For $\lambda=\left[21^{n-2}\right]$ there can be at most one pair of type $\mathrm{C}$ in a matching; the rules for such a pair are precisely those given in parts $2 \mathrm{a}(\mathrm{i})$ and $2 \mathrm{~b}(\mathrm{i})$ of Definition 5.7. The above rules are again a restatement of the remaining parts of the algorithm in this case.

Although we have not been able to find a general rule for pairs of type C, some guidance is offered by the following observations for two extreme situations when a pair of type $\mathrm{C}$ is examined:

- If all pairs of $\delta$ previously examined are between-class pairs, then the pair is treated by the rules for type B given above. This follows from the requirement that the conjectured statistic is 0 precisely for bipartite matchings. 
- If all pairs of $\delta$ previously examined contribute to the statistic and there are no completed cycles yet in $G\left(\delta, \delta_{\lambda}\right)$, then if the pair terminates a cycle in $G\left(\delta, \delta_{\lambda}\right)$ of length less than $n=|\lambda|$, it does not contribute to the statistic. This follows from part 2 of Proposition 3.6.

With the aid of these observations we have found additional algorithms that do indeed explain some of the computed data but we have not proved that they hold for any infinite subclass. Perhaps the lack of obvious symmetry between $\mu$ and $\nu$ in the procedure suggested above accounts for the proliferation of cases in these algorithms.

We do not know of a convenient class of nonbipartite matchings $\delta$ of cardinality $(n-1)$ ! such that $\Lambda(\delta, \varepsilon)=\mu$ and $\Lambda\left(\delta, \delta_{\lambda}\right)=\nu$ but in view of part 2 of Proposition 3.6 this might be helpful in finding statistics in general.

\section{THE HYPERMAP SERIES AND POSITIVITY CONJECTURES}

We conclude by returning briefly to the hypermap series $h_{\mu \nu}^{\lambda}(b)$ that, from (4), is given by

$$
\Psi(\mathbf{x}, \mathbf{y}, \mathbf{z} ; t, 1+b)=1+\sum_{n \geq 1} t^{n} \sum_{\lambda, \mu, \nu \vdash n} h_{\mu \nu}^{\lambda}(b) p_{\lambda}(\mathbf{x}) p_{\mu}(\mathbf{y}) p_{\nu}(\mathbf{z}) .
$$

From the properties of Jack symmetric functions, the hypermap series $h_{\mu \nu}^{\lambda}(b)$ is a rational function of $b$ for all $\lambda, \mu, \nu \vdash n$. Moreover, from Theorem 1.1 we can immediately relate it to the hypermap coefficients defined in the Introduction.

Proposition 6.1. For $\lambda, \mu, \nu \vdash n$,

$$
\text { 1) } \begin{aligned}
h_{\mu \nu}^{\lambda}(0) & =m_{\mu \nu}^{\lambda}, \\
\text { 2) } h_{\mu \nu}^{\lambda}(1) & =l_{\mu \nu}^{\lambda} .
\end{aligned}
$$

Table 2 gives $h_{\mu \nu}^{\lambda}(b)$ for maps with at most 3 edges (so all parts of $\lambda$ are equal to 2). To make the table compact, use has been made of the duality $h_{\mu \nu}^{\lambda}(b)=h_{\nu \mu}^{\lambda}(b)$. In each case that is given the entries are nonnegative integer polynomials in $b$, and we conjecture this to be the case in general.

Conjecture 6.2. For all $\lambda, \mu, \nu \vdash n \geq 1$,

$$
h_{\mu \nu}^{\lambda}(b) \text { is a nonnegative integer polynomial in } b \text {. }
$$

In view of the combinatorial context in which the specializations to $b=0,1$ arose we further conjecture that $h_{\mu \nu}^{\lambda}(b)$ is a generating series for hypermaps with respect to a combinatorial statistic $\vartheta$ associated with maps.

Conjecture 6.3 (Hypermap-Jack Conjecture). For $\lambda, \mu, \nu \vdash n$,

$$
h_{\mu \nu}^{\lambda}(b)=\sum b^{\vartheta(M)}
$$

where the sum is over all hypermaps $M$ in locally orientable surfaces with hyperedge distribution $\lambda$, vertex distribution $\mu$ and face distribution $\nu$, and $\vartheta(M)$ is a nonnegative integer which equals 0 if and only if $M$ is orientable.

The Matchings-Jack Conjecture does not imply the Hypermap-Jack Conjecture directly since $c_{\mu \nu}^{\lambda}(b)$ is divided by $(1+b)^{l(\lambda)}$ in $(5)$ in $\Phi$, the logarithm is taken, 
TABLE 2. The hypermap series $h_{\mu \nu}^{\lambda}(b)$ for maps with at most 3 edges

\begin{tabular}{|c|c|c|c|c|c|c|c|}
\hline edge $\lambda$ & vertex $\mu$ & face $\nu$ & $h_{\mu \nu}^{\lambda}(b)$ & edge $\lambda$ & vertex $\mu$ & face $\nu$ & $h_{\mu \nu}^{\lambda}(b)$ \\
\hline$[2]$ & {$[2]$} & {$[2]$} & $b$ & \multirow[t]{14}{*}[2^{3}]{} & {$[6]$} & {$\left[31^{3}\right]$} & 2 \\
\hline & {$[2]$} & {$\left[1^{2}\right]$} & 1 & & {$[6]$} & {$\left[2^{3}\right]$} & $b$ \\
\hline \multirow[t]{6}{*}[2^{2}]{} & {$[4]$} & {$[4]$} & $1+b+3 b^{2}$ & & {$[6]$} & {$\left[2^{2} 1^{2}\right]$} & 3 \\
\hline & {$[4]$} & {$\left[\begin{array}{ll}3 & 1\end{array}\right]$} & $4 b$ & & {$\left[\begin{array}{ll}5 & 1\end{array}\right]$} & {$\left[\begin{array}{ll}5 & 1\end{array}\right]$} & $18 b$ \\
\hline & {$[4]$} & {$\left[2^{2}\right]$} & $b$ & & {$\left[\begin{array}{ll}5 & 1\end{array}\right]$} & {$[42]$} & $6 b$ \\
\hline & {$[4]$} & {$\left[21^{2}\right]$} & 2 & & {$\left[\begin{array}{ll}5 & 1\end{array}\right]$} & {$\left[41^{2}\right]$} & 6 \\
\hline & {$\left[\begin{array}{ll}3 & 1\end{array}\right]$} & {$\left[\begin{array}{ll}3 & 1\end{array}\right]$} & 4 & & {$\left[\begin{array}{ll}5 & 1\end{array}\right]$} & {$\left[3^{2}\right]$} & $6 b$ \\
\hline & {$\left[2^{2}\right]$} & {$\left[2^{2}\right]$} & 1 & & {$\left[\begin{array}{ll}5 & 1\end{array}\right]$} & {$\left[\begin{array}{lll}3 & 2 & 1\end{array}\right]$} & 6 \\
\hline \multirow[t]{6}{*}[2^{3}]{} & {$[6]$} & {$[6]$} & $13 b+13 b^{2}+15 b^{3}$ & & {$\left[\begin{array}{ll}4 & 2\end{array}\right]$} & {$[42]$} & $6 b$ \\
\hline & {$[6]$} & {$\left[\begin{array}{ll}5 & 1\end{array}\right]$} & $6+6 b+18 b^{2}$ & & {$[42]$} & {$\left[3^{2}\right]$} & $3 b$ \\
\hline & $6]$ & {$\left[\begin{array}{ll}42 \\
{[}\end{array}\right]$} & $3+3 b+9 b^{2}$ & & {$[42]$} & {$\left[\begin{array}{lll}3 & 2 & 1\end{array}\right]$} & 6 \\
\hline & [6] & {$\left[41^{2}\right]$} & $9 b$ & & {$\left[41^{2}\right]$} & {$\left[3^{2}\right]$} & 3 \\
\hline & {$[6]$} & {$\left[3^{2}\right]$} & $1+b+5 b^{2}$ & & {$\left[3^{2}\right]$} & {$\left[2^{3}\right]$} & 1 \\
\hline & {$[6]$} & {$\left[\begin{array}{lll}3 & 2 & 1\end{array}\right]$} & $12 b$ & & & & \\
\hline
\end{tabular}

and this is then multiplied by $1+b$ to obtain $\Psi$. However, the conjectures are combinatorially related, since, from the matchings representation of a hypermap given in [6], some canonical form of each connected component of the matchings graph $G\left(\varepsilon, \delta_{\lambda}, \delta\right)$ considered in Sections 4 and 5 above corresponds to a hypermap.

From a study of our data, certain facts and conjectures have emerged. First, if the Hypermap-Jack Conjecture is true, then the combinatorial statistic $\vartheta$ depends on the rooting of a hypermap. This can be seen, for example, by considering maps with 2 edges, a single vertex and a single face. Table 2 gives $h_{[4],[4]}^{\left[2^{2}\right]}(b)=1+b+3 b^{2}$, so there are 5 such rooted maps in all. It is readily checked that these correspond to 3 unrooted maps. One of the unrooted maps has one rooting, and is orientable, and each of the other two unrooted maps have two distinct rootings. The latter two pairs of rooted maps must therefore account for the terms $b+3 b^{2}$. It follows that for one of these pairs, different rootings of the same map give different values (namely 1 and 2) of the statistic $\vartheta$. Our computations suggest that $\vartheta$ is a statistic that is positively correlated with departure of a map from orientability.

Second, the degree of the connection series $h_{\mu \nu}^{\lambda}(b)$ appears to be $n-l(\lambda)-l(\mu)-$ $l(\nu)+2$, where $\lambda, \mu, \nu \vdash n$, a much more precise result than Proposition 3.6 for $c_{\mu \nu}^{\lambda}(b)$. Further, the coefficient of this biggest power of $b$ appears to be $\left|\mathcal{C}_{\nu}\right|$ in the case $\lambda=\mu=[n]$ (and the other cases that correspond by symmetry). We do not know of a convenient class of nonorientable hypermaps of this cardinality, but this might be a fruitful case to consider. Finally, it seems from the data that $h_{\mu \nu}^{\lambda}(b)$ is unimodal.

\section{ACKNOWLEDGEMENTS}

This research was supported by individual operating grants from the Natural Sciences and Engineering Research Council of Canada. We thank Luis Goddyn, Phil Hanlon, Richard Stanley and John Stembridge for helpful discussions and suggestions. We thank John Stembridge also for assistance in using his SF package. 


\section{REFERENCES}

1. M. Adler and P. van Moerbeke, Birkhoff strata, Backlund transformations, and regularization of isospectral operators, Advances in Math., 108 (1994), 140-204. CMP 95:01

2. G.E. Andrews, I.P. Goulden and D.M. Jackson, Generalizations of Cauchy's summation theorem for Schur functions, Trans. Amer. Math. Soc., 310 (1988), 805-820. MR 89m:05009

3. N. Bergeron and A.M. Garsia, Zonal polynomials and domino tableaux, Discrete Math., 99 (1992), 3-15. MR 93e:20006

4. I.P. Goulden and D.M. Jackson, "Combinatorial Enumeration", Wiley Interscience, New York, 1983. MR 84m:05002

5. I.P. Goulden and D.M. Jackson, Combinatorial constructions for integrals over normally distributed random matrices, Proc. Amer. Math. Soc., 123 (1995), 995-1003. CMP 95:06

6. I.P. Goulden and D.M. Jackson, Maps in locally orientable surfaces, the double coset algebra, and zonal polynomials, Canadian J. Math. (to appear).

7. I.P. Goulden and D.M. Jackson, Symmetric functions and Macdonald's result for top connexion coefficients in the symmetric group, J. Algebra, 166 (1994), 364-378. CMP 94:13

8. P.J. Hanlon, Jack symmetric functions and some combinatorial properties of Young symmetrizers, J. Combinatorial Theory (A), 47 (1988), 37-70. MR 90e:05008

9. P.J. Hanlon, A Markov chain on the symmetric group and Jack symmetric functions, Discrete Math., 99 (1992), 123-140. MR 93j:60093

10. P.J. Hanlon, R.P. Stanley, and J.R. Stembridge, Some combinatorial aspects of the spectra of normally distributed random matrices, Contemporary Mathematics, 138 (1992), 151-174. MR 93j:05164

11. H. Jack, A class of symmetric polynomials with a parameter, Proc. Roy. Soc. Edinburgh (A), 69 (1969-70), 1-18. MR 44:6652

12. D.M. Jackson and T.I. Visentin, A character theoretic approach to embeddings of rooted maps in an orientable surface of given genus, Trans. Amer. Math. Soc., 322 (1990), 343-363. MR 91b:05093

13. D.M. Jackson and T.I. Visentin, Character theory and rooted maps in an orientable surface of given genus: face-coloured maps, Trans. Amer. Math. Soc., 322 (1990), 365-376. MR 91b:05094

14. K.J. Kadell, The Selberg-Jack symmetric functions, (preprint).

15. I.G. Macdonald, "Symmetric functions and Hall polynomials," Clarendon Press, Oxford, 1979. MR 84g:05003

16. I.G. Macdonald, Commuting differential operators and zonal spherical functions, in "Algebraic Groups, Utrecht 1986" (A.M. Cohen, et al., Editors), Lecture Notes in Math., 1271 189-200, Springer-Verlag, Berlin, 1987. MR 89e:43025

17. I.G. Macdonald, A new class of symmetric functions, Publ. I.R.M.A. Strasbourg, Actes 20th Seminaire Lotharingien (1988), 131-171.

18. R.P. Stanley, Some combinatorial properties of Jack symmetric functions, Advances in Math., 77 (1989), 76-115. MR 90g:05020

19. J.R. Stembridge, A Maple package for symmetric functions - Version 2, Department of Mathematics, University of Michigan, Ann Arbor, MI 48109, July 1993.

Department Combinatorics and Optimization, University of Waterloo, Waterloo,

ONTARIO N2L 3G1, CANADA

E-mail address: ipgoulden@math.uwaterloo.ca

E-mail address: dmjackson@watdragon.uwaterloo.ca 